\title{
RANDOM PRODUCTS OF CONTRACTIONS IN BANACH SPACES
}

\author{
J. DYE, M. A. KHAMSI, AND S. REICH
}

\begin{abstract}
We show that the random product of a finite number of $(W)$ contractions converges weakly in all smooth reflexive Banach spaces. If one of the contractions is compact, then the convergence is uniform.
\end{abstract}

Let $(X,|\cdot|)$ be a (real) Banach space and $T: X \rightarrow X$ a linear operator. Recall that $T$ is called a contraction if $|T v| \leq|v|$ for all vectors $v$ in $X$. We say that a contraction $T$ satisfies condition $(W)$ if whenever $\left\{v_{n}\right\}$ is bounded and $\left|v_{n}\right|-\left|T v_{n}\right| \rightarrow 0$, it follows that the weak $\lim _{n \rightarrow \infty}\left(v_{n}-T v_{n}\right)=0$. The algebraic semigroup $\mathbf{S}$ generated by a (possibly infinite) set of contractions will be said to satisfy condition $(W)$ if for any bounded sequence of vectors $\left\{v_{n}\right\} \subset X$ and a sequence of words $\left\{W_{n}\right\}$ from $\mathbf{S}$ such that $\left|v_{n}\right|-\left|W_{n} v_{n}\right| \rightarrow 0$, the weak $\lim _{n \rightarrow \infty}\left(v_{n}-W_{n} v_{n}\right)=0$.

Now let $\left\{T_{1}, T_{2}, \ldots\right\}$ be a sequence of contractions that satisfy condition $(W)$, and let $r$ be a self-mapping of the set of natural numbers. A random product of these contractions is the sequence $\left\{S_{n}: n=1,2, \ldots\right\}$ defined by

$$
S_{n}=T_{r(n)} T_{r(n-1)} \cdots T_{r(1)} .
$$

Our purpose in this paper is to study the convergence properties of such products. Such a study is not only of intrinsic interest, but is also motivated by applications to the numerical solution of partial differential equations $[2,14]$ and linear inequalities [19], approximation theory [16, 9] and computer tomography $[18,11]$.

We begin by noting several properties of contractions which satisfy condition $(W)$. We shall call such contractions $(W)$ contractions for short. We then consider the algebraic semigroup generated by a finite number of $(W)$ contractions and establish a weak convergence theorem for random products of these contractions in all smooth reflexive Banach spaces (Theorem 1). This seems to be the first such result outside Hilbert space. It includes the Hilbert space theorem due to Amemiya and Ando [1]. We continue with a weak convergence result for semigroups generated by an infinite sequence of contractions (Theorem 2). Finally, we obtain a uniform convergence theorem for random

Received by the editors October 24, 1988 and, in revised form, April 4, 1989.

1980 Mathematics Subject Classification (1985 Revision). Primary 47A05; Secondary 47B05. $65 \mathrm{~J} 10$.

Key words and phrases. Contraction, random product, weak convergence. 
products of compact contractions (Theorem 3). We first observe (cf. [1]) the following fact.

Lemma 1. The class of $(W)$ contractions is closed under composition.

Proof. Let $T_{1}$ and $T_{2}$ satisfy condition $(W)$ and suppose that $\left\{v_{n}\right\}$ is bounded and $\left|v_{n}\right|-\left|T_{2} T_{1} v_{n}\right| \rightarrow 0$. Since $\left|T_{2} T_{1} v_{n}\right| \leq\left|T_{1} v_{n}\right| \leq\left|v_{n}\right|$, we also have $\left|v_{n}\right|-\left|T_{1} v_{n}\right| \rightarrow 0$ and $\left|T_{1} v_{n}\right|-\left|T_{2} T_{1} v_{n}\right| \rightarrow 0$. Hence

the weak $\lim _{n \rightarrow \infty}\left(v_{n}-T_{1} v_{n}\right)=$ the weak $\lim _{n \rightarrow \infty}\left(T_{1} v_{n}-T_{2} T_{1} v_{n}\right)=0$.

The result follows.

We denote the range of an operator $T$ by $R(T)$ and its fixed point set by $F(T)$. In the sequel we shall repeatedly use the following simple observation: If $\left\{v_{n}\right\}$ is a bounded sequence of vectors in a reflexive Banach space $X$ which does not converge weakly to 0 , then there is a subsequence $\left\{w_{k}\right\}$ of $\left\{v_{n}\right\}$ such that $w_{k}=u+q_{k}$, where $u \neq 0$ and $\left\{q_{k}\right\}$ converges weakly to 0 .

Proposition 1. If $X$ is a reflexive Banach space and the contraction $T: X \rightarrow X$ satisfies $(W)$, then for each $x$ in $X,\left\{T^{n} x\right\}$ converges weakly to a fixed point of $T$.

Proof. Recall that the mean ergodic theorem provides us with a contractive projection $Q: X \rightarrow F(T)$ such that $T Q=Q T=Q$. the sequence Therefore we may restrict our attention to $x$ in $R(I-Q)$, a closed subspace of $X$ which is invariant under $T$. If $\left\{T^{n} x\right\}$ does not converge weakly to 0 , then there is a subsequence of $\left\{T^{n} x\right\}$, which we denote by $x_{k}$, such that $x_{k}=u+q_{k}$, where $u \neq 0$ and $\left\{q_{k}\right\} \rightarrow 0$ weakly. Since $T$ is a $(W)$ contraction, we have $\lim _{n \rightarrow \infty}\left|T^{n} x\right|=\lim _{n \rightarrow \infty}\left|T^{n+1} x\right|$ and the weak $\lim _{n \rightarrow \infty}\left(T^{n} x-T^{n+1} x\right)=0$. Hence $0=$ the weak $\lim _{n \rightarrow \infty}\left(x_{k}-T x_{k}\right)=$ the weak $\lim _{n \rightarrow \infty}\left(u-T u+q_{k}-T q_{k}\right)=$ $u-T u$. Thus $u$ belongs to $F(T) \cap R(I-Q)=\{0\}$. The contradiction we have reached shows that $\left\{T^{n} x\right\}$ does converge weakly to 0 and the proof is complete.

Alternatively, we note that for any $x$ in $X$ and $k \geq 0$,

$$
\begin{aligned}
\left|\left(\sum_{i=1}^{n} T^{i+k} x\right) / n-Q x\right| & =\left|\left(\sum_{i=1}^{n} T^{i+k} x-T^{k} Q x\right) / n\right| \\
& \leq\left|\left(\sum_{i=1}^{n} T^{i} x\right) / n-Q x\right| .
\end{aligned}
$$

Therefore the strong $\lim _{n \rightarrow \infty}\left(\sum_{i=1}^{n} T^{i} x\right) / n=Q x$, uniformly in $k \geq 0$. But we also know that the weak $\lim _{n \rightarrow \infty}\left(T^{n} x-T^{n+1} x\right)=0$ and this is a Tauberian condition for almost convergence. Hence $\left\{T^{n} x\right\}$ converges weakly to $Q x$.

In order to proceed we need the following simple lemma (cf. [1]).

Lemma 2. If $\left\{T_{j}: 1 \leq j \leq N\right\}$ are $(W)$ contractions, then

$$
\bigcap\left\{F\left(T_{j}\right): 1 \leq j \leq N\right\}=F\left(T_{N} T_{N-1} \cdots T_{1}\right) .
$$


Proof. It is clear that $\bigcap\left\{F\left(T_{j}\right): 1 \leq j \leq N\right\} \subset F\left(T_{N} T_{N-1} \cdots T_{1}\right)$. Conversely, suppose $x \in F\left(T_{N} T_{N-1} \cdots T_{1}\right)$ and let $y=T_{N-1} \cdots T_{1} x$. Then $|x|=\left|T_{N} y\right| \leq$ $|y| \leq|x|$. Since $T_{N}$ satisfies condition $(W), y=T_{N} y$. Hence $T_{N-1} \cdots T_{1} x=$ $x, T_{N} x=x$, and the lemma follows.

Our next result is obtained by combining Proposition 1 with Lemmas 1 and 2.

Proposition 2. Let $\left\{T_{j}: 1 \leq j \leq N\right\}$ be $N(W)$ contractions on a reflexive Banach space $X$. Then the weak $\lim _{n \rightarrow \infty}\left(T_{N} T_{N-1} \cdots T_{1}\right)^{n} x=Q x$ exists for all $x$ in $X$ and defines a contractive projection $Q$ of $X$ onto $\bigcap\left\{F\left(T_{j}\right): 1 \leq j \leq\right.$ $N\}$.

It is known [17, Lemma 3.2;15, p. 162] that if $E$ is a closed subspace of a smooth Banach space $X$, then there is at most one nonexpansive retraction of $X$ onto $E$. In the setting of Proposition 2 this means that if $X$ is also smooth, then the contractive projection $Q$ is the only nonexpansive retraction of $X$ onto $\bigcap\left\{F\left(T_{j}\right): 1 \leq j \leq N\right\}$. Since $Q T_{j}$ is also such a retraction, it follows that $Q T_{j}=Q=T_{j} Q$ for each $1 \leq j \leq N$, and that $X$ is the direct sum of the closed subspaces $R(Q)$ and $R(I-Q)$, both of which are invariant under each $T_{j}, 1 \leq j \leq N$.

We say that a contraction $T$ satisfies condition $\left(W^{\prime}\right)$ if $|v|=|T v|$ implies that $v=T v$. (See [10].) Clearly a $(W)$ contraction also satisfies condition $\left(W^{\prime}\right)$. It turns out that in some spaces these two conditions are equivalent.

Let $\varphi: R^{+} \rightarrow R^{+}$be continuous and strictly increasing, with $\varphi(0)=0$ and $\lim _{t \rightarrow \infty} \varphi(t)=\infty$. Recall that the duality map $J_{\varphi}$ from a Banach space $X$ into the family of nonempty (by the Hahn-Banach Theorem) subsets of its dual $X^{*}$, corresponding to the gauge function $\varphi$, is defined by

$$
J_{\varphi}(x)=\left\{x^{*} \in X^{*}:\left(x, x^{*}\right)=|x|\left|x^{*}\right| \text { and }\left|x^{*}\right|=\varphi(|x|)\right\} .
$$

This duality map is single-valued if $X$ is smooth. It is said to be weakly sequentially continuous if $X$ is smooth and whenever the sequence $\left\{x_{n}\right\}$ converges weakly to $x,\left\{J_{\varphi}\left(x_{n}\right)\right\}$ converges weak-star to $J_{\varphi}(x)$. This is the case, for example, for all $l_{p}$ spaces, $1<p<\infty$.

Proposition 3. If the reflexive Banach space has a weakly sequentially continuous duality map, then the conditions $(W)$ and $\left(W^{\prime}\right)$ are equivalent.

Proof. Let $T: X \rightarrow X$ be a contraction that satisfies condition $\left(W^{\prime}\right)$, and assume that $\left\{v_{n}\right\} \subset X$ is a bounded sequence such that $\left|v_{n}\right|-\left|T v_{n}\right| \rightarrow 0$. If $\left\{v_{n}-T v_{n}\right\}$ does not converge weakly to 0 , then $\left\{v_{n}\right\}$ has a subsequence, which we continue to denote by $\left\{v_{n}\right\}$, that converges weakly to $u \notin F(T)$. We may also assume that $\lim _{n \rightarrow \infty}\left|v_{n}\right|$ exists. We write $v_{n}=u+q_{n}$, where $q_{n} \rightarrow 0$ weakly. Now let $J_{\varphi}: X \rightarrow X^{*}$ be a weakly sequentially continuous duality map with a gauge function $\varphi: R^{+} \rightarrow R^{+}$, which is continuous and strictly increasing, with $\varphi(0)=0$ and $\lim _{t \rightarrow \infty} \varphi(t)=\infty$, and set $\Phi(t)=\int_{0}^{t} \varphi(r) d r$. 
Since $\Phi(|x+y|)=\Phi(|x|)+\int_{0}^{1}\left(y, J_{\varphi}(x+t y)\right) d t$ for all $x$ and $y$ in $X$, we have

$$
\Phi\left(\left|u+q_{n}\right|\right)=\Phi\left(\left|q_{n}\right|\right)+\int_{0}^{1}\left(u, J_{\varphi}\left(q_{n}+t u\right)\right) d t
$$

and

$$
\Phi\left(\left|T u+T q_{n}\right|\right)=\Phi\left(\left|T q_{n}\right|\right)+\int_{0}^{1}\left(T u, J_{\varphi}\left(T q_{n}+t T u\right)\right) d t
$$

Therefore,

$$
\begin{aligned}
\int_{0}^{1}\left(u, J_{\varphi}(t u)\right) d t-\int_{0}^{1}\left(T u, J_{\varphi}(t T u)\right) d t & \\
= & \lim _{n \rightarrow \infty}\left[\int_{0}^{1}\left(u, J_{\varphi}\left(q_{n}+t u\right)\right) d t-\int_{0}^{1}\left(T u, J_{\varphi}\left(T q_{n}+t T u\right)\right) d t\right] \\
\leq & \lim _{n \rightarrow \infty}\left[\int_{0}^{1}\left(u, J_{\varphi}\left(q_{n}+t u\right)\right) d t-\int_{0}^{1}\left(T u, J_{\varphi}\left(T q_{n}+t T u\right)\right) d t\right. \\
& \left.+\Phi\left(\left|q_{n}\right|\right)-\Phi\left(\left|T q_{n}\right|\right)\right] \\
= & \lim _{n \rightarrow \infty}\left[\Phi\left(\left|u+q_{n}\right|\right)-\Phi\left(\left|T u+T q_{n}\right|\right)\right]=0 .
\end{aligned}
$$

In other words,

$$
|u| \int_{0}^{1} \varphi(|t u|) d t \leq|T u| \int_{0}^{1} \varphi(t|T u|) d t
$$

or $\Phi(|u|) \leq \Phi(|T u|)$. Hence $|T u|=|u|$ and $T u=u$ by condition $\left(W^{\prime}\right)$. This contradiction completes the proof.

Combining Propositions 1 and 3 we obtain the following improvement of [5, Corollary 8.1].

Proposition 4. If $X$ is a reflexive Banach space with a weakly sequentially continuous duality map and the contraction $T: X \rightarrow X$ satisfies condition $\left(W^{\prime}\right)$, then for each $x$ in $X,\left\{T^{n} x\right\}$ converges weakly to a fixed point of $T$.

Conditions $(W)$ and $\left(W^{\prime}\right)$ are not equivalent in all reflexive Banach spaces. To see this, consider the space $l^{2}$ renormed by $|x|=\max \left\{|x|_{2}, 2^{1 / 2}|x|_{\infty}\right\}$ and the contraction $T$ defined by $(T x)_{n}=a_{n} x_{n}$, where $0 \leq a_{n}<1$ and $\left\{a_{n}\right\}$ increases to 1 . Set $v_{n}=e_{1}+e_{n}$ where $e_{n}$ is the $n$th unit vector. Then $\left(\left|v_{n}\right|-\left|T v_{n}\right|\right) \rightarrow 0$, but the weak $\lim _{n \rightarrow \infty}\left(v_{n}-T v_{n}\right) \neq 0$. On the other hand, there are Banach spaces (such as the $l_{p}$ sum of a sequence of finite-dimensional nonsmooth spaces) which do not have a weakly sequentially continuous duality map, but in which conditions $(W)$ and $\left(W^{\prime}\right)$ are equivalent. To see this, note that what is required in the proof of Proposition 3 is the following property of a Banach space $X$ (cf. [13, p. 137]):

If $\left|q_{n}\right| \rightarrow s$ and $q_{n} \rightarrow 0$ weakly, then $\lim _{n \rightarrow \infty}\left|u+q_{n}\right|=\delta(|u|, s)$ for all $u$ in $X$, where $\delta: R^{+} \times R^{+} \rightarrow R^{+}$is strictly increasing in the first variable 
and increasing in the second. (In the case of Proposition 3, $\delta(r, s)=$ $\Phi^{-1}(\Phi(r)+\Phi(s))$. $)$

We remark in passing that Propositions 1 and 2 have nonlinear analogs. Let $C$ be a closed convex subset of a Banach space $X$. Recall that an operator $T: C \rightarrow C$ is said to be nonexpansive if $|T x-T y| \leq|x-y|$ for all $x$ and $y$ in $C$. We say that a nonexpansive mapping $T$ satisfies condition $(W)$ if whenever $\left\{x_{n}-y_{n}\right\}$ is bounded and $\left(\left|x_{n}-y_{n}\right|-\left|T x_{n}-T y_{n}\right|\right) \rightarrow 0$ it follows that the weak $\lim _{n \rightarrow \infty}\left(\left(x_{n}-y_{n}\right)-\left(T x_{n}-T y_{n}\right)\right)=0$.

Proposition 5. Let $\left\{T_{j}: 1 \leq j \leq N\right\}$ be $N$ nonexpansive self-mappings of a closed convex bounded subset $C$ of a Banach space $X$. If each $T_{j}$ satisfies condition $(W)$ and $X$ is uniformly convex with a Fréchet differentiable norm, then the weak $\lim _{n \rightarrow \infty}\left(T_{N} T_{N-1} \cdots T_{1}\right)^{n} x=Q x$ exists for all $x$ in $C$ and defines a nonexpansive retraction of $C$ onto $\bigcap\left\{F\left(T_{j}\right): 1 \leq j \leq N\right\}$.

Proof. Let $y$ be a fixed point of $T=T_{N} T_{N-1} \cdots T_{1}$. Then

$$
\left(\left|T^{n} x-y\right|-\left|T^{n+1} x-y\right|\right) \rightarrow 0 .
$$

Since $T$ also satisfies condition $(W)$, the weak $\lim _{n \rightarrow \infty}\left(T^{n} x-T^{n+1} x\right)=0$. Since this is a Tauberian condition for weak almost convergence, the result is now seen to follow from the nonlinear mean ergodic theorem.

Since all strongly nonexpansive mappings in the sense of [6] satisfy condition $(W)$, we see that in a uniformly convex space all averaged and firmly nonexpansive mappings (including all linear contractive projections and all resolvents of accretive operators) satisfy condition $(W)$. We also note that all weakly compact convex subsets of Banach spaces have the fixed point property for nonexpansive mapping which satisfy condition $(W)$.

We now turn out attention to semigroups.

Proposition 6. Let $\left\{T_{j}: 1 \leq j \leq N\right\}$ be $N(W)$ contractions on a smooth reflexive Banach space. Then the algebraic semigroup $\mathbf{S}=\mathbf{S}\left(T_{1}, T_{2}, \ldots, T_{N}\right)$ generated by them also satisfies condition $(W)$.

Proof. Let $Q_{k}$ be the unique contractive projection of the Banach space $X$ onto $\bigcap\left\{F\left(T_{j}\right): 1 \leq j \leq k\right\}, 1 \leq k \leq N$, which is provided by Proposition 2 and the remarks following it. In the sequel we shall decompose the $v_{n}$ 's which appear in the definition of condition $(W)$ as $v_{n}^{(1)}+v_{n}^{(2)}$, where $v_{n}^{(1)} \in Q_{k} X$ and $v_{n}^{(2)} \in\left(I-Q_{k}\right) X$, for an appropriate $k$.

We proceed by induction, considering the case $N=1$ first. For some bounded $v_{n}$ and some self-mapping $k(n)$ of the set of natural numbers for which

$$
\left|v_{n}\right|-\left|T^{k(n)} v_{n}\right| \rightarrow 0
$$

we wish to show that $v_{n}-T^{k(n)} v_{n} \rightarrow 0$ weakly. Since $T v_{n}^{(1)}=v_{n}^{(1)}$, it suffices to show that

$$
\left(I-T^{k(n)}\right) v_{n}^{(2)} \rightarrow 0 \text { weakly. }
$$


We can drop to a subsequence and assume $k(n)>1$. In light of (1), we have $\left|v_{n}\right|-\left|T v_{n}\right| \rightarrow 0$, which implies that

$$
v_{n}^{(2)}-T v_{n}^{(2)} \rightarrow 0 \text { weakly, }
$$

as $T$ has $(W)$. If $v_{n}^{(2)}$ does not converge weakly to zero, we may assume for a subsequence that $v_{n}^{(2)}=u+q_{n}$, where $u \neq 0$ and $q_{n} \rightarrow 0$ weakly. Hence (3) becomes

$$
u+q_{n}-T u-T q_{n} \rightarrow 0 \text { weakly. }
$$

The closed subspace $(I-Q) X$ (where $\left.Q=Q_{1}\right)$ is invariant for $T$, and includes its weak limits. Hence, $u \in(I-Q) X$. But (4) implies $T u=u$. We conclude that $u=0$, and this contradiction implies that

$$
v_{n}^{(2)} \rightarrow 0 \text { weakly. }
$$

Using (1) again, we see that $\left|T^{k(n)-1} v_{n}\right|-\left|T^{k(n)} v_{n}\right| \rightarrow 0$, which implies that $T^{k(n)-1} v_{n}-T^{k(n)} v_{n} \rightarrow 0$ weakly. Hence

$$
T^{k(n)-1} v_{n}^{(2)}-T^{k(n)} v_{n}^{(2)} \rightarrow 0 \text { weakly. }
$$

If $T^{k(n)-1} v_{n}^{(2)}$ does not converge weakly to zero, then for a subsequence, $T^{k(n)-1} v_{n}^{(2)}=u+q_{n}$, where $u \neq 0$ and $q_{n} \rightarrow 0$ weakly. Then (6) becomes

$$
u+q_{n}-T u-T q_{n} \rightarrow 0 \text { weakly. }
$$

As before, (7) implies that $T u=u$, which implies that $u=0$, and this contradiction implies that $T^{k(n)} v_{n}^{(2)} \rightarrow 0$ weakly. This fact and (5) imply that $v_{n}^{(2)}-T^{k(n)} v_{n}^{(2)} \rightarrow 0$ weakly. We conclude that $v_{n}-T^{k(n)} v_{n} \rightarrow 0$ weakly.

Proceeding inductively, suppose the Proposition is valid for words from the first $k-1<N$ letters. Say there exist bounded vectors $v_{n}$ and words $W_{n} \in$ $S\left(T_{1}, \ldots, T_{k}\right)$ such that

$$
\left|v_{n}\right|-\left|W_{n} v_{n}\right| \rightarrow 0
$$

We wish to show that $v_{n}^{(2)}-W_{n} v_{n}^{(2)} \rightarrow 0$ weakly. (Here $v_{n}^{(2)} \in\left(I-Q_{k}\right) X$.) By induction and a possible drop to a subsequence, we may further assume that the $W_{n}$ 's are complete (each contains all $k$ letters). Suppose $v_{n}^{(2)}$ does not converge weakly to zero. We may drop to a subsequence and assume

$$
v_{n}^{(2)}=u+q_{n} \text {, where } u \neq 0 \text { and } q_{n} \rightarrow 0 \text { weakly. }
$$

An easy combinatorial argument (and a possible reindexing of the $T_{j}$ 's, $j=$ $1, \ldots, k)$ allows us to assume that (for some subsequence) there exist words $F_{n}$ and $T_{k}$ such that $W_{n}=(\cdots) T_{k} F_{n}$, where the $F_{n} \in \mathbf{S}\left(T_{1}, \ldots, T_{k-1}\right)$ and are complete (in $T_{1}, \ldots, T_{k-1}$ ).

Our immediate goal is to show that $u$ is fixed by the $F_{n}$. If $u$ is not fixed, note the first letters $T_{n}$ in $F_{n}$ for which $u$ is not a fixed point. Denote by $A_{n}$ 
the interceding words (which fix $u$ ). (In the event the word $A_{n}$ is null, replace it with the identity map $I$.) Since we are dealing with just $k-1$ distinct maps, we may assume by dropping to a subsequence that $T_{k} F_{n}=(\cdots) T A_{n}$, for some fixed map $T$. Note that (8) implies that $\left|v_{n}\right|-\left|A_{n} v_{n}\right| \rightarrow 0$. Hence by induction and (9) we have

$$
u+q_{n}-A_{n} u-A_{n} q_{n}=q_{n}-A_{n} q_{n} \rightarrow 0 \text { weakly, }
$$

which implies that

$$
A_{n} q_{n} \rightarrow 0 \text { weakly. }
$$

By (9) and the fact that $T$ has $(W)$ we have

$$
u+q_{n}-T A_{n} u-T A_{n} q_{n}=u-T u+q_{n}-T A_{n} q_{n} \rightarrow 0 \text { weakly. }
$$

Hence (11) implies that $u=T u$, a contradiction. Thus $F_{n} u=u$.

By (8), $\left|F_{n} v_{n}\right|-\left|T_{k} F_{n} v_{n}\right| \rightarrow 0$. As $T_{k}$ has $(W)$, we must have that $F_{n} v_{n}-$ $T_{k} F_{n} v_{n} \rightarrow 0$ weakly. This implies that $F_{n} v_{n}^{(2)}-T_{k} F_{n} v_{n}^{(2)} \rightarrow 0$ weakly. Using (9), we have $u+F_{n} q_{n}-T_{k} u-T_{k} F_{n} q_{n} \rightarrow 0$ weakly. Since $F_{n}=A_{n}$, we have $F_{n} q_{n} \rightarrow 0$ weakly and $T_{k} F_{n} q_{n} \rightarrow 0$ weakly by (11). Hence $u$ is fixed by $T_{k}$, as well as the other $k-1$ maps. This means that $u=0$, as $u \in\left(I-Q_{k}\right) X$. We conclude that

$$
v_{n}^{(2)} \rightarrow 0 \text { weakly. }
$$

We will be done if we show that $W_{n} v_{n}^{(2)} \rightarrow 0$ weakly. Suppose that $W_{n} v_{n}^{(2)}$ does not converge weakly to zero. Then by dropping to a subsequence we may assume that $W_{n} v_{n}^{(2)} \rightarrow z \neq 0$, weakly. In a similar manner as before, a combinatorial argument (and a possible reindexing of the $T_{j}$ 's, $j=1, \ldots, k$ ) allows us to assume that (for some subsequence) there exist words $F_{n} T_{k}$ such that $W_{n}=F_{n} T_{k}(\cdots)$, where the $F_{n} \in \mathbf{S}\left(T_{1}, \ldots, T_{k-1}\right)$ and are complete (in $\left.T_{1}, \ldots, T_{k-1}\right)$. Suppose $(\cdots) v_{n}^{(2)}$ does not converge weakly to zero. We drop to a subsequence for which

$$
(\cdots) v_{n}^{(2)}=u+q_{n} \text {, where } u \neq 0 \text { and } q_{n} \rightarrow 0 \text { weakly. }
$$

By (8), $\left|(\cdots) v_{n}\right|-\left|T_{k}(\cdots) v_{n}\right| \rightarrow 0$. Since $T_{k}$ has $(W)$, we have that $(\cdots) v_{n}-$ $T_{k}(\cdots) v_{n} \rightarrow 0$ weakly. Hence $(\cdots) v_{n}^{(2)}-T_{k}(\cdots) v_{n}^{(2)} \rightarrow 0$ weakly. In light of (14), we have $u+q_{n}-T_{k} u-T_{k} q_{n} \rightarrow 0$ weakly. So $u$ is fixed by $T_{k}$. Our goal, as before, is to show that $u$ is fixed by all the other $k-1$ maps. Let $A_{n}$ and $T$ be defined as before in the proof. Now $\left|T_{k}(\cdots) v_{n}\right|-\left|A_{n} T_{k}(\cdots) v_{n}\right| \rightarrow$ 0 , so by induction we have $T_{k}(\cdots) v_{n}-A_{n} T_{k}(\cdots) v_{n} \rightarrow 0$ weakly, and hence $T_{k}(\cdots) v_{n}^{(2)}-A_{n} T_{k}(\cdots) v_{n}^{(2)} \rightarrow 0$ weakly. Substituting into (14), we have $u+$ $T_{k} q_{n}-A_{n} u-A_{n} T_{k} q_{n} \rightarrow 0$ weakly. This implies that

$$
A_{n} T_{k} q_{n} \rightarrow 0 \text { weakly. }
$$

Now $T$ has $(W)$, so that, using (8), we have $u+A_{n} T_{k} q_{n}-T u-T A_{n} T_{k} q_{n} \rightarrow$ 0 weakly. By (15) we see that $u$ is necessarily fixed by $T$. Recalling that 
$u \in\left(I-Q_{k}\right) X$, we must have that $u=0$, a contradiction. Hence $(\cdots) v_{n}^{(2)} \rightarrow 0$ weakly. But (15) and $u=0$ imply that $A_{n} T_{k}(\cdots) v_{n}^{(2)} \rightarrow 0$ weakly. Clearly $T_{k}(\cdots) v_{n}^{(2)} \rightarrow 0$ weakly, because $u=0$ in (14). Hence $W_{n} v_{n}^{(2)} \rightarrow 0$ weakly, a contradiction, as $W_{n} v_{n}^{(2)} \rightarrow z \neq 0$. Hence $W_{n} v_{n}^{(2)} \rightarrow 0$ weakly, completing the proof.

Remark. Inspection of the proof shows that if $\left|v_{n}\right|-\left|W_{n} v_{n}\right| \rightarrow 0$ and the $W_{n}$ are complete, them in fact both $v_{n}^{(2)}$ and $W_{n} v_{n}^{(2)}$ converge weakly to zero.

Theorem 1. Let $\left\{T_{j}: 1 \leq j \leq N\right\}$ be $N(W)$ contractions on a smooth reflexive Banach space $X$. Let $r$ be a mapping of the set of natural numbers onto $\{1,2, \ldots, N\}$ which assumes each value infinitely often and let $S_{n}=$ $T_{r(n)} T_{r(n-1)} \cdots T_{r(1)}$. Then the weak $\lim _{n \rightarrow \infty} S_{n} x=Q x$ exists for each $x$ in $X$ and $Q$ is the unique contractive projection of $X$ onto $\bigcap\left\{F\left(T_{j}\right): 1 \leq j \leq N\right\}$.

Proof. It suffices to show that $S_{n} x \rightarrow 0$ weakly for $x \in(I-Q) X$. If $S_{n} x$ does not converge weakly to zero, then by dropping to a subsequence (which we continue to denote by $S_{n}$ ), we may assume $S_{n} x=u+q_{n}$, where $u \neq 0$ and $q_{n} \rightarrow 0$ weakly. Since $u \in(I-Q) X$, and each map occurs infinitely often, we may note the first letters $T_{n}$ in the original sequence (succeeding the last letter in $S_{n}$ ) for which $u$ is not a fixed point. Denote by $A_{n}$ the interceding words (which fix $u$ ). (In the event the word $A_{n}$ is null, replace it with the identity map $I$.) Since we are dealing with just $N$ distinct maps, we may assume by dropping to a subsequence that $T_{n} A_{n} S_{n}=T A_{n} S_{n}$, for some fixed map $T \in\left\{T_{1}, \ldots, T_{N}\right\}$. Since we have $\left|S_{n} x\right|-\left|A_{n} S_{n} x\right| \rightarrow 0$, Proposition 6 enables us to conclude that $\left(I-A_{n}\right) S_{n} x=q_{n}-A_{n} q_{n} \rightarrow 0$ weakly. Hence $A_{n} q_{n} \rightarrow 0$ weakly. We also have $\left|A_{n} S_{n} x\right|-\left|T A_{n} S_{n} x\right| \rightarrow 0$. Since $T$ has $(W)$, we have that

$$
(I-T) A_{n} S_{n} x=u+A_{n} q_{n}-T u-T A_{n} q_{n} \rightarrow 0 \text { weakly. }
$$

Since $T A_{n} q_{n} \rightarrow 0$ weakly, we see that $u=T u$. This contradiction completes the proof.

Alternatively, for any $n$, let $C_{n}$ be a complete word immediately following $S_{n}$. These exist as each map occurs infinitely often. If $x \in(I-Q) X$, then $S_{n} x \in(I-Q) X$, by the remarks following Proposition 2. $\mathbf{S}\left(T_{1}, \ldots, T_{N}\right)$ has $(W)$, by Proposition 6. Hence $\left|S_{n} x\right|-\left|C_{n} S_{n} x\right| \rightarrow 0$ implies $S_{n} x \rightarrow 0$ weakly, as the $C_{n}$ are complete. (Here we have substituted $S_{n} x$ for $v_{n}^{(2)}$ and applied the remark at the end of the proof of Proposition 6.)

Remark. Using Theorem 1.11 on p. 80 of [12], we may replace smoothness by the assumption that the adjoints $\left\{T_{j}^{*}: 1 \leq j \leq N\right\}$ are also $(W)$ contractions.

We now present a weak convergence theorem for random products of an infinite sequence of contractions. For other results on such products see $[2,4$, and 8]. One may note that the random product of an infinite sequence of $(W)$ contractions need not converge weakly. See [4, p. 330; 7]. 
Theorem 2. Let $\left\{T_{1}, T_{2}, \ldots\right\}$ be contractions on a reflexive Banach space $X$ such that the algebraic semigroup $\mathbf{S}=\mathbf{S}\left(T_{1}, T_{2}, \ldots\right)$ has $(W)$. Let $r$ be a mapping of the set of natural numbers into itself with the property that each range value which is assumed, is assumed infinitely often, and let $S_{n}=T_{r(n)} T_{r(n-1)} \cdots$ $T_{r(1)}$. Then the weak $\lim _{n \rightarrow \infty} S_{n} x=P x$ exists for each $x$ in $X$ and $P$ is $a$ contractive projection of $X$ onto $\bigcap_{n=1}^{\infty}\left\{F\left(T_{r(n)}\right)\right\}$.

Proof. Suppose there exist subsequences $\{n\}$ and $\left\{n^{\prime}\right\}$ such that $S_{n} x \rightarrow u$ weakly and $S_{n^{\prime}} x \rightarrow u^{\prime}$ weakly. Select a subsequence $\left\{n^{\prime \prime}\right\}$ of $\{n\}$ such that there exists an element of $\left\{n^{\prime}\right\}$ between any two consecutive elements of $\left\{n^{\prime \prime}\right\}$. For this new subsequence $S_{n^{\prime \prime}}$, let $W_{n}$ denote a (possibly null) word separating, in the original sequence, $S_{n^{\prime \prime}}$ from the very next occurrence of an element of $\left\{n^{\prime}\right\}$. Hence, for an appropriate subsequence of $\left\{n^{\prime}\right\}$, we may assume that $S_{n^{\prime}} x=W_{n} S_{n^{\prime \prime}} x$. Clearly $\left|S_{n^{\prime \prime}} x\right|-\left|W_{n} S_{n^{\prime \prime}} x\right| \rightarrow 0$. Now $(W)$ for $\mathbf{S}$ implies that $S_{n^{\prime \prime}} x-W_{n} S_{n^{\prime \prime}} x \rightarrow 0$ weakly. As $\left\{n^{\prime \prime}\right\}$ is a subsequence of $\{n\}$, we have that $S_{n^{\prime \prime}} x \rightarrow u$ weakly. Thus $u=u^{\prime}$. We conclude that the original sequence converges to $u$ weakly. If $u \notin \bigcap_{n=1}^{\infty}\left\{F\left(T_{r(n)}\right)\right\}$, and each map occurs infinitely often, there exists a fixed map $T$ such that $T u \neq u$, and a subsequence $S_{n} x$ with the property that the very next map in the original sequence is $T$. We have $\left|S_{n} x\right|-\left|T S_{n} x\right| \rightarrow 0$. This implies that $T u=u$ as $T$ has $(W)$. This contradiction completes the proof.

Remark. If $\left\{T_{1}, T_{2}, \ldots\right\}$ is a sequence of $(W)$ contractions on a smooth reflexive Banach space $X$, then there still exists a unique contractive projection onto $\bigcap\left\{F\left(T_{j}\right): 1 \leq j<\infty\right\}$. This is because the intersection is a nonexpansive retract by [3, Lemma 4] and the retraction onto it is unique (and, in fact, linear) by [17]. Alternatively, we may show directly that if $X$ is reflexive and $T_{j}: X \rightarrow X, 1 \leq j<\infty$ are $\left(W^{\prime}\right)$ contractions, then there is a $\left(W^{\prime}\right)$ contractive projection onto $\bigcap\left\{F\left(T_{j}\right): 1 \leq j<\infty\right\}$. These projections coincide with the projection $P$ obtained in Theorem 2 if $X$ is smooth.

In the setting of Theorem 1, condition $(W)$ is not strong enough to guarantee strong convergence (see [8]). We do, however, obtain uniform convergence if one of the $T_{j}$ 's is compact. First we need two lemmas.

Recall that a Banach space is said to have the Kadec-Klee property (KKP for short) if whenever $x_{n} \rightarrow x$ weakly with $\left|x_{n}\right| \rightarrow|x|$, it follows that $x_{n} \rightarrow x$ strongly. We say that a contraction satisfies condition $(S)$ if whenever the sequence $\left\{v_{n}\right\}$ is bounded and $\left|v_{n}\right|-\left|T v_{n}\right| \rightarrow 0$, it follows that the strong $\lim _{n \rightarrow \infty}(I-T) v_{n}=0$.

Lemma 3. For a compact contraction $T$ on a reflexive Banach space $X$, the conditions $\left(W^{\prime}\right)$ and $(W)$ are equivalent. In the event $X$ also has the $K K P$, they are also equivalent to condition $(S)$.

Proof. We always have $(S) \Rightarrow(W) \Rightarrow\left(W^{\prime}\right)$. So it suffices to show that $\left(W^{\prime}\right) \Rightarrow$ $(W)(\Rightarrow(S)$ if $X$ has the KKP). 
Let $\left\{v_{n}\right\} \subset X$ be a bounded sequence such that $\left|v_{n}\right|-\left|T v_{n}\right| \rightarrow 0$. If $\left\{v_{n}-\right.$ $\left.T v_{n}\right\}$ fails to converge weakly to 0 , then $\left\{v_{n}\right\}$ has a subsequence, which we continue to denote by $\left\{v_{n}\right\}$, which converges weakly to $u \notin F(T)$. We may also assume that $s=\lim _{n \rightarrow \infty}\left|v_{n}\right|$ exists. Since $T$ is compact, $\left\{T v_{n}\right\}$ converges strongly to $T u$ and

$$
|T u|=\lim _{n \rightarrow \infty}\left|T v_{n}\right|=\lim _{n \rightarrow \infty}\left|v_{n}\right|=s
$$

But we also have

$$
s=|T u| \leq|u| \leq \lim _{n \rightarrow \infty} \inf \left|v_{n}\right|=s .
$$

Hence $|u|=|T u|$ and $u=T u$ by condition $\left(W^{\prime}\right)$. This contradiction proves that $\left(W^{\prime}\right) \Rightarrow(W)$.

If $X$ also has the $\mathrm{KKP}$, then $\left\{v_{n}\right\}$ converges strongly to $u$ because $|u|=$ $\lim _{n \rightarrow \infty}\left|v_{n}\right|$. Thus the strong $\lim _{n \rightarrow \infty}(I-T) v_{n}=0$ and the result follows.

Our next lemma generalizes the following observation.

Let $X$ be a reflexive Banach space and let $\left\{T_{1}, \ldots, T_{N}\right\}$ be $N$ contractions on $X$, each satisfying $\left(W^{\prime}\right)$, which have no common nonzero fixed points. Assume at least one of the $T_{j}$ 's is compact and let $T=T_{1} \cdots T_{N}$. Then $\|T\|<1$.

Indeed, if we let $L C(X)$ denote all compact operators on $X$, then since $L C(X)$ is an ideal in $L(X), T \in L C(X)$. An argument similar to the proof of Lemma 1 shows that $T$ satisfies $\left(W^{\prime}\right)$. Since the norm is a continuous function and the image of the unit ball is compact, there exists an $x$ with $|x|=1$ such that $\|T\| \quad\left(=\sup _{|y| \leq 1}|T y|\right)=|T x|$. In any event, $\|T\| \leq 1$. If $\|T\|=1$, then $|T x|=|x|$. Thus, by $\left(W^{\prime}\right), T x=x$. By Lemma 2, $x \in \bigcap_{i=1}^{N} F\left(T_{i}\right)$. Since by assumption the $T_{j}$ 's have no common nonzero fixed points, this rules out $\|T\|=1$, proving $\|T\|<1$.

Lemma 4. Let $\left\{T_{1}, \ldots, T_{N}\right\}$ be $N$ contractions on a smooth reflexive $B a$ nach space satisfying condition $\left(W^{\prime}\right)$. Assume $T_{1}$ is cornpact. Let $\left\{W_{n}\right\}$ be a sequence of complete words in the $T_{i}$. Let $Q$ be the projection onto $\bigcap\left\{F\left(T_{j}\right): 1 \leq j \leq N\right\}$. Then, on $(I-Q) X$, $\sup _{n}\left\|W_{n}\right\|<1$.

Proof. If $\sup _{n}\left\|W_{n}\right\|=1$ on $(I-Q) X$, there exist $x_{n}$ of norm one such that $\lim _{n}\left|W_{n} x_{n}\right|=1$. Hence $\left|x_{n}\right|-\left|W_{n} x_{n}\right| \rightarrow 0$. Since $x_{n} \in(I-Q) X$, and the $\left\{T_{j}: 1 \leq j \leq N\right\}$ have $(W)$ by Lemma 3 , we may apply the remark following Proposition 6, and conclude that

$$
W_{n} x_{n} \rightarrow 0 \text { weakly. }
$$

We factor $W_{n}$ as $W_{n}=U_{n} T_{1} V_{n}$.

As $T_{1}$ is compact, we can assume $T_{1} V_{n} x_{n} \rightarrow u \neq 0$ by dropping to a subsequence. Some first letter $T$ in $U_{n}$ (following $T_{1}$ ) moving $u$ must occur infinitely often, as each map occurs infinitely often. If not, we would have $W_{n} x_{n} \rightarrow u$ strongly, contradicting (16). Letting $A_{n}$ denote the separating 
words, we have that

$$
T A_{n} T_{1} V_{n} x_{n} \rightarrow T u \text { weakly }
$$

and

$$
A_{n} T_{1} V_{n} x_{n} \rightarrow u \text { weakly. }
$$

Clearly $\left|A_{n} T_{1} V_{n} x_{n}\right|-\left|T A_{n} T_{1} V_{n} x_{n}\right| \rightarrow 0$. By $(W)$ for $T$, we must have that $T u=u$. This contradicts the construction of $T$, and finishes the proof.

Theorem 3. Let $\left\{T_{1}, \ldots, T_{N}\right\}$ be contractions on a smooth reflexive Banach space $X$, each satisfying condition $\left(W^{\prime}\right)$. Assume one of these operators is compact. Let $r$ be a mapping from the natural numbers onto $\{1, \ldots, N\}$ which assumes each value infinitely often, and let $S_{n}=T_{r(n)} \cdots T_{r(1)}$. Then $S_{n}$ converges uniformly to the projection $Q$ on the subspace $\bigcap\left\{F\left(T_{i}\right): 1 \leq i \leq N\right\}$. Proof. We can assume $\left\|T_{i}\right\|=1$ for each $i$, for otherwise $S_{n}$ will converge uniformly to 0 , as each map occurs infinitely often. Now by the remarks following Proposition 2, the projection $Q$ commutes with each $T_{i}$. Moreover, we have $S_{n} Q=Q S_{n}=Q$. Since the product of $\left(W^{\prime}\right)$ operators is a $\left(W^{\prime}\right)$ operator, the operators $(I-Q) T_{i}=T_{i}(I-Q), 1 \leq i \leq N$, are contractions on $(I-Q) X$ satisfying $\left(W^{\prime}\right)$, having (as we can assume) norm 1 , and having no common nonzero fixed points. Since $r$ assumes each value infinitely often, we can find a sequence $W_{n}=(I-Q) T_{r(k(n)+t(n))} T_{r(k(n)+t(n)-1)} \cdots T_{r(k(n))}$ of complete words in the $(I-Q) T_{i}$ whose index sets $\{k(n), \ldots, k(n)+t(n)\}$ are mutually disjoint. Since one of the operators $(I-Q) T_{i}$ is compact, Lemma 4 provides us with a positive constant $M$ such that $\left\|W_{n}\right\| \leq M<1$; for all $n$. Thus, if $m$ is chosen so large that $S_{m}$ contains $k$ of the words $W_{n}$, then $\left\|(I-Q) S_{m}\right\| \leq M^{k}$. Thus $\left\|S_{m}-Q\right\|=\left\|(I-Q) S_{m}\right\| \leq M^{k}$, which goes to 0 as $m$ (and therefore $k$ ) goes to $\infty$, proving the theorem.

This theorem improves upon the corresponding Hilbert space result in [7].

Remark. As mentioned earlier, condition $(W)$ on each of $N$ maps is not sufficient for strong convergence of random products. It can be shown that even the assumption of $(S)$ on just two maps will not allow the conclusion of strong convergence of all random products, even when $X$ is Hilbert. Hence an additional condition on the maps, such as compactness (as in Theorem 3 ), is needed. In this connection, see also [4].

We conclude this paper with an analog of Proposition 6.

Proposition 7. Let $\left\{T_{j}: 1 \leq j \leq N\right\}$ be $N$ ( $\left.W^{\prime}\right)$ compact contractions on a smooth reflexive Banach space $X$ with the $K K P$. Then the algebraic semigroup $\mathbf{S}=\mathbf{S}\left(T_{1}, T_{2}, \ldots, T_{N}\right)$ generated by them satisfies condition $(S)$.

Proof. Suppose there exist bounded $v_{n}$ such that $\left|v_{n}\right|-\left|W_{n} v_{n}\right| \rightarrow 0$ yet $\left(I-W_{n}\right) v_{n}$ does not converge strongly to zero. Then for a subsequence we may assume that $\left|\left(I-W_{n}\right) v_{n}\right|>\varepsilon>0$. By Lemma 3 and Proposition 6 we know that

$$
\left(I-W_{n}\right) v_{n} \rightarrow 0 \text { weakly. }
$$


Since $X$ is reflexive, we may assume

$$
v_{n} \rightarrow u \text { weakly }
$$

for a further subsequence. Hence (17) implies that

$$
W_{n} v_{n} \rightarrow u \text { weakly, }
$$

also.

Since there are only a finite number of maps, some fixed map $T$ occurs infinitely often as a first letter of $W_{n}$. By dropping to a further subsequence, we may assume that $T$ always follows $v_{n}$ and that $T v_{n}$ converges strongly. In light of (18), $T v_{n} \rightarrow T u$ (strongly). Since $v_{n}-T v_{n} \rightarrow 0$ strongly because $T$ has $(S)$, we see that $v_{n} \rightarrow T u$ strongly. Now (18) implies that $T u=u$. Hence $v_{n} \rightarrow u$ strongly. By dropping to an appropriate further subsequence, we may assume $W_{n}$ always ends in the same (compact) map, and that $W_{n} v_{n}$ converges strongly. Necessarily $W_{n} v_{n} \rightarrow u$ strongly, because of (19). This contradicts our initial assumption that $\left|\left(I-W_{n}\right) v_{n}\right|>\varepsilon>0$, completing the proof.

Acknowledgments. The first author was partially supported by a California State University, Northridge Grant for Faculty Research and Creative Activity. The third author was partially supported by the Fund for the Promotion of Research at the Technion. All the authors thank the referee for several helpful suggestions.

\section{REFERENCES}

1. I. Amemiya and T. Ando, Convergence of random products of contractions in Hilbert space, Acta Sci. Math. (Szeged) 26 (1965), 239-244.

2. F. E. Browder, On some approximation methods for solutions of the Dirichlet problem for linear elliptic equations of arbitrary order, J. Math. Mech. 7 (1958), 69-80.

3. R. E. Bruck, Properties of fixed-point sets of nonexpansive mappings in Banach spaces, Trans. Amer. Math. Soc. 179 (1973), 251-262.

4. Random products of contractions in metric and Banach spaces, J. Math. Anal. Appl. 88 (1982), 319-332.

5. __ Asymptotic behavior of nonexpansive mappings, Contemp. Math. 18 (1983), 1-47.

6. R. E. Bruck and S. Reich, Nonexpansive projections and resolvents of accretive operators in Banach spaces, Houston J. Math. 3 (1977), 459-470.

7. J. Dye, Convergence of random products of compact contractions in Hilbert space, Integral Equations Operator Theory 12 (1989), 12-22.

8. A products of contractions in Hilbert space, Integral Equations Operator Theory 12 (1989), 155-162.

9. C. Franchetti and W. Light, The alternating algorithm in uniformly convex spaces, J. London Math. Soc. 29 (1984), 545-555.

10. I. Halperin, The product of projection operators, Acta Sci. Math. (Szeged) 23 (1962), 96-99.

11. C. Hamaker and D. C. Solmon, The angles between the null spaces of X-rays, J. Math. Anal. Appl. 62 (1978), 1-23.

12. U. Krengel, Ergodic theorems, De Gruyter, Berlin, 1985.

13. T C. Lim, Asymptotic centers and nonexpansive mappings in conjugate Banach spaces, Pacific J. Math. 90 (1980), 135-143. 
14. P. L. Lions, On the Schwarz alternating method. I, Domain Decomposition Methods for Partial Differential Equations, SIAM, Philadelphia, Pa., 1988, pp. 1-42.

15. S. Reich, Product formulas, nonlinear semigroups and accretive operators, J. Funct. Anal. 36 (1980), 147-168.

16. _ Nonlinear semigroups, accretive operators and applications, Nonlinear Phenomena in Mathematical Sciences, Academic Press, New York, 1982, pp. 831-838.

17. __ A limit theorem for projections, Linear and Multilinear Algebra 13 (1983), 281-290.

18. K. T. Smith, D. C. Solmon, and S. L. Wagner, Practical and mathematical aspects of reconstructing objects from radiographs, Bull. Amer. Math. Soc. 83 (1977), 1227-1270.

19. J. E. Spingarn, A projection method for least-squares solutions to over-determined systems of linear inequalities, Linear Algebra Appl. 86 (1987), 211-236.

Department of Mathematics, California State University, Northridge, California 91330

Department of Mathematics, University of Rhode Island, Kingston, Rhode Island 02881-0816

Department of Mathematics, University of Southern California, Los Angeles, CaliFORNIA 90089

Department of Mathematics, Technion-The Israel Institute of Technology, Haifa 32000, ISRAEL 